This is the accepted version of the following article:

Fuertes-Espinosa C., García-Simón C., Pujals M., Garcia-Borràs M., Gómez L., Parella T., Juanhuix J., I maz I., Maspoch D., Costas M., Ribas X.. Supramolecular Fullerene Sponges as Catalytic Masks for Regioselective Functionalization of C60. Chem, (2020). 6. : 169 - . 10.1016/j.chempr.2019.10.010,

which has been published in final form at https://dx.doi.org/10.1016/j.chempr.2019.10.010 (c) https://dx.doi.org/10.1016/j.chempr.2019.10.010. This manuscript version is made available under the CC-BY-NC-ND 4.0 license http://creativecommons. org/licenses/by-nc-nd/4.0/ 


\title{
Supramolecular Fullerene Sponges as Catalytic Masks for Regioselective Functionalization of $\mathbf{C}_{60}$
}

\author{
Carles Fuertes-Espinosa, ${ }^{[a]}$ Cristina García-Simón, ${ }^{[a]}$ Míriam Pujals, ${ }^{[a]}$ Marc Garcia- \\ Borràs, ${ }^{[a]}$ Laura Gómez, ${ }^{[b]}$ Teodor Parella, ${ }^{[c]}$ Judit Juanhuix, ${ }^{[\mathrm{d}]}$ Inhar Imaz, ${ }^{[\mathrm{e}]}$ Daniel \\ Maspoch, ${ }^{[\mathrm{e}, \mathrm{f}]}$ Miquel Costas, ${ }^{[\mathrm{a}]}$ and Xavi Ribas ${ }^{[\mathrm{a}] *}$
}

[a] Institut de Química Computacional i Catàlisi and Departament de Química, Universitat de Girona, Campus Montilivi, E-17003 Girona (Catalonia, Spain). E-mail: xavi.ribas@udg.edu

[b] Serveis Tècnics de Recerca (STR), Universitat de Girona, Parc Científic i Tecnològic, E17003 Girona (Catalonia, Spain)

[c] Servei de RMN and Departament de Química, Facultat de Ciències, Universitat Autònoma de Barcelona (UAB), Campus UAB, E-08193 Bellaterra (Catalonia, Spain)

[d] ALBA Synchrotron, E-08290 Cerdanyola del Vallès, Barcelona.(Catalonia, Spain)

[e] Catalan Institute of Nanoscience and Nanotechnology (ICN2), CSIC and the Barcelona Institute of Science and Technology, Campus UAB, E-08193 Bellaterra, Barcelona (Catalonia, Spain)

[f] ICREA, Pg. Lluís Companys 23, E-08010 Barcelona (Catalonia, Spain)

\begin{abstract}
Isomer-pure poly-functionalizalized fullerenes are required to boost the development of fullerene chemistry in any field, and specifically in solar cell design. ${ }^{[1-3]}$ Easy-accessible monofunctionalized fullerenes are mainly used in organic- or perovskite-based solar cells, ${ }^{[4-7]}$ due to the hampered accessibility to pure-isomer poly-adduct derivatives. In a general basis, multi-adduct mixtures with uncontrolled regioselectivity are obtained, and chromatographic purification is prohibitively costly and time-consuming to be used in the production of solar cells. Moreover,
\end{abstract}


single isomer poly-functionalized fullerenes are only accessible via stoichiometric, multistep paths entailing protecting-unprotecting sequences. ${ }^{[8-14]}$ Herein, a nanocapsule is used as a supramolecular mask to exert full control on the reactivity and the equatorial regioselectivity of Bingel-Hirsch cyclopropanation reactions of confined fullerene guests. Thus, equatorial bis-, trisand tetrakis- $\mathrm{C}_{60}$ homo-adducts are exclusively obtained in a stepwise manner, completely avoiding over functionalization (always observed in bulk solution) and holding precise control on the number of additions. Regioselectivity is strictly dictated by the four cross-shaped open windows in the tetragonal prismatic supramolecular container. ${ }^{[15]}$ Furthermore, isomer-pure equatorial hetero-tetrakis-adducts or hetero-Th-hexakis-adducts are synthesized at will in one-pot synthesis for the first time. Remarkably, a synthetically useful biphasic protocol is designed to achieve selective and quantitative formation of exclusively tetrakis-adducts using catalytic amounts of nanocapsule. Therefore, the confinement control imposed by the capsule's cavity conforms a unique practical strategy to infer regio-control to the synthesis of fullerene equatorial poly-adducts. This work provides a synthetically valuable path to produce a plethora of new pure-isomer polyfunctionalized $\mathrm{C}_{60}$-based compounds, as candidates for testing in solar cell devices and for biomedical applications. ${ }^{[16-17]}$

\section{Main Text}

From the early stages of their discovery, fullerene species have gained increasing interest in several fields including biomedical chemistry, ${ }^{[16-19]}$ material science, ${ }^{[20]}$ solar energy conversion ${ }^{[7]}$ and energy storage. ${ }^{[21]} \mathrm{A}$ variety of synthetic routes concerning the functionalization of fullerenes have been established to improve their solubility and to modify their physicochemical properties. ${ }^{[22-23]}$ To date, $\mathrm{C}_{60}$ fullerene, which has a highly symmetric structure and can be produced in large-scale, has been the most exploited substrate. In 2013, $\mathrm{C}_{60}$ fullerene and its derivatives were introduced into Perovskites Solar Cells (PSCs), demonstrating that their use in the perovskite layers plays an important role in passivating the charge traps at the surface and grain boundaries of the thin film, leading to higher device performance (power conversion efficiencies (PCE) above 20\%). ${ }^{[24]}$ Easyaccessible mono-adduct $\mathrm{PC}_{60} \mathrm{BM}$ (and also $\mathrm{PC}_{70} \mathrm{BM}$ ) are mainly used as electron transport layers (ETLs) in solar cell devices. ${ }^{[2,4]}$ It is generally accepted that the regio-controlled multiple functionalization is the Achilles' heel of fullerene derivative preparation, which heavily impacts 
in many fields, and solar cells in particular. ${ }^{[5,7]}$ This was clearly shown by Grätzel in the enhanced PCE $(20.8 \%)$ and better stability of PSCs when pure $\alpha$-bis-PC ${ }_{60} \mathrm{BM}$ was utilized in the generation of the perovskite thin layer, compared to standard mono-functionalized $\mathrm{PC}_{60} \mathrm{BM}(19.9 \%) .{ }^{[1]}$ In line with this, Imahori clearly demonstrated the detrimental use of regioisomer mixtures compared to pure isomers in organic photovoltaics (OPVs). ${ }^{[3]}$ Therefore, synthetic access to novel, precisely poly-functionalized fullerenes is needed to unravel structure-property relationships and drive the design of improved solar cell devices. ${ }^{[6]}$ Generally, the spherical nature of $\mathrm{C}_{60}$ containing several double bonds with almost identical reactivity, compromises the controlled synthesis of siteselective $^{[25]}$ and regioselective ${ }^{[10]}$ multiple adducts of these species. Thus, purification of polyfunctionalized fullerene mixtures usually involves tedious and time-consuming chromatographic separation protocols, hampering the full exploitation of these derivatives. To address this shortcoming, different strategies for the selective production of multiple substituted fullerenes have been pursued, generally involving the initial stoichiometric modification of the fullerene with directing groups. Highlighted are the "tether-directed remote functionalization" strategy for the synthesis of bis-, tris-, pentakis- and hexakis-adducts under regiocontrol, firstly developed by Diederich in 1994 (Fig. 1a), ${ }^{[8,11,13-14,26]}$ and the "orthogonal transposition" synthetic strategy developed by Kräutler in 1997 (Fig. 1b), ${ }^{[12]}$ for the production of equatorial cyclopropanated tetrakis-diethylmalonate- $\mathrm{C}_{60}$ adduct (Bingel-Hirsch reaction), which is used for further functionalization on the available axial positions. ${ }^{[9]}$ On the other hand, the modulation of the $\mathrm{C}_{60}$ regiochemical reactivity by confinement in supramolecular receptors remains scarce. Cubic ${ }^{[27]}$ and a bowl-shaped ${ }^{[28]}$ coordination cages and a metal-organic-framework ${ }^{[29]}$ had been used as supramolecular hosts, all limited to Diels-Alder reactions and leading to formation of mono or bisadducts. Arguably, all methods for regioselective functionalization of fullerenes reported to date, [27, 30-31] albeit elegant, are tedious, limited in scope, and overall lack of the versatility and practical utility required to boost the applicability of fullerenes. In a broad sense, the regioselective polyfunctionalization of fullerenes showcases the general problem of exerting selectivity in a large spherical-like molecule, a challenging question in organic synthesis.

Here we present an unprecedented straightforward supramolecular mask strategy to prepare exclusively equatorial bis-, tris- and tetrakis-cyclopropanated- $\mathrm{C}_{60}$ Bingel-Hirsch derivatives. By taking advantage of the high affinity of porphyrin-based tetragonal prismatic supramolecular nanocapsules $\left(\mathrm{Pd}-\right.$ based 1a· $(\mathrm{BArF})_{8}$ and $\mathrm{Cu}$-based $\left.\mathbf{1 b} \cdot(\mathrm{BArF})_{8}\right)$ for fullerenes, ${ }^{\left[15,{ }^{32]}\right.}{ }^{\text {highly stable }}$ 
$\mathrm{C}_{60} \subset \mathbf{1 a} \cdot(\mathrm{BArF})_{8}$ host-guest complex is submitted to Bingel-Hirsch cyclopropanation reaction conditions. Regioselectivity is strictly dictated by the four cross-shaped apertures of the supramolecular tetragonal prismatic container in a controlled fashion. Moreover, the usually observed over-reactivity that commonly drives to the formation of poly-adducts is cancelled upon confinement of $\mathrm{C}_{60}$, and clean, stepwise cyclopropanated adducts up to tetrakis-additions are obtained in excellent yields and purities. 
a.

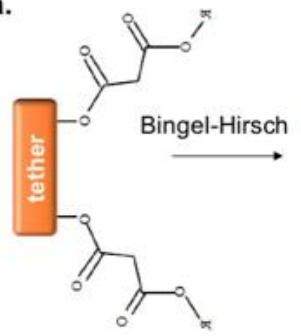

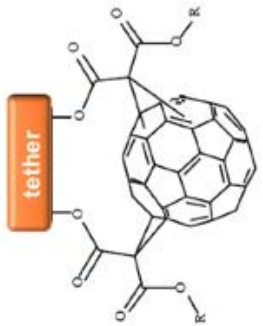

b.

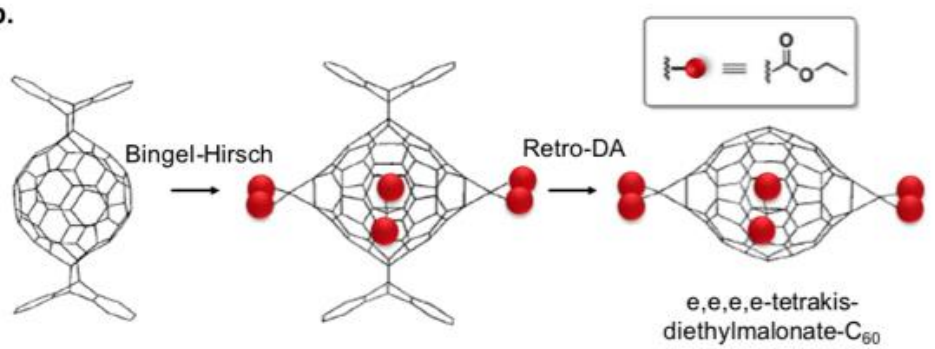

c. Supramolecular mask strategy (this work)
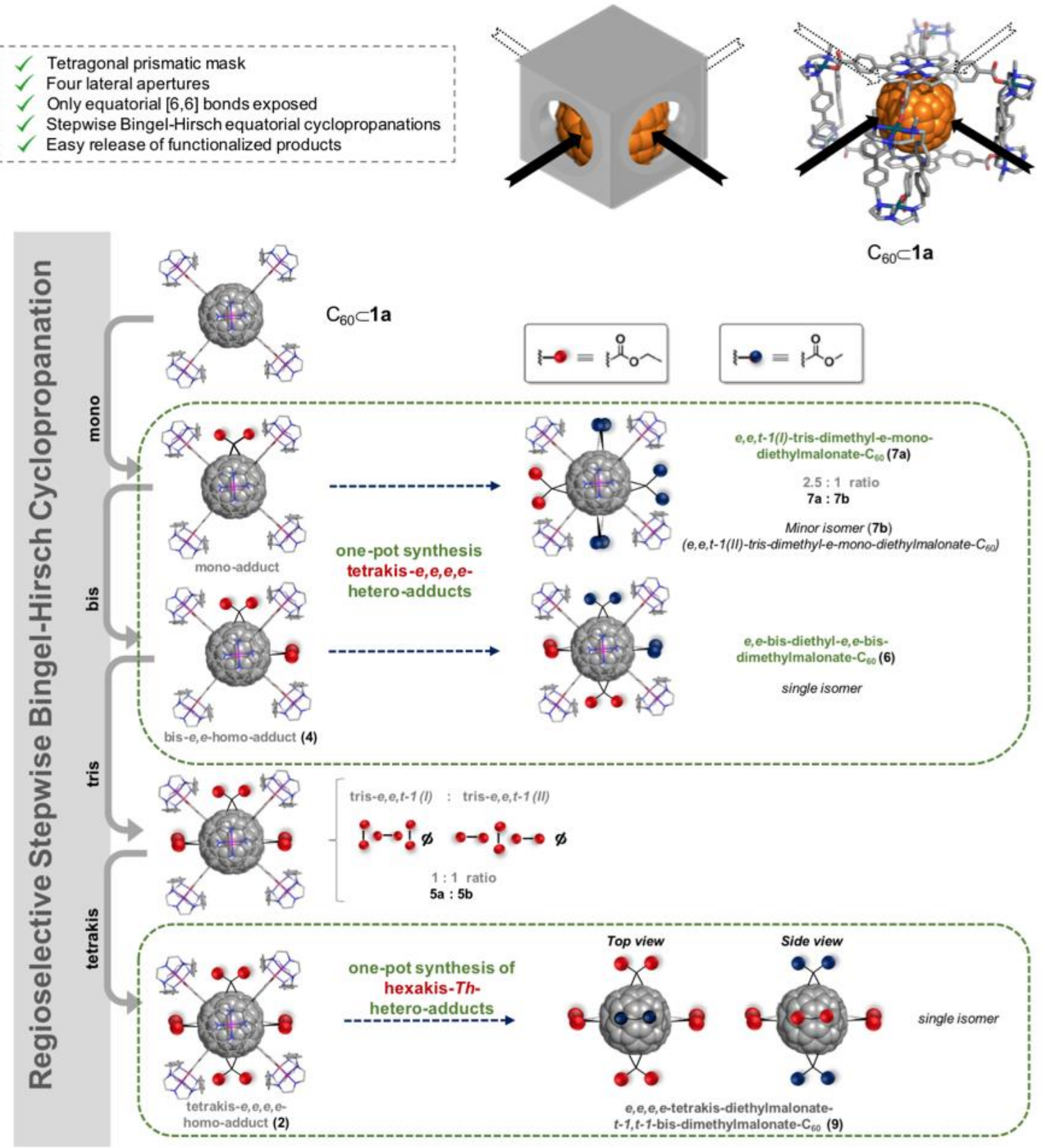

Fig. 1. Synthetic strategies aiming at regioselective functionalization of $\mathbf{C}_{60}$. a) tether-directed bis-adduct functionalization; $\mathbf{b}$ ) topochemically controlled orthogonal transposition to obtain fully equatorial tetrakis-adducts, involving Dields-Alder (DA), 
Bingel-Hirsch and retro-DA steps; c) supramolecular mask strategy (this work) using encapsulated $\mathrm{C}_{60}$ in tetragonal prismatic nanocapsule $\mathbf{1 a} \cdot(\mathrm{BArF})_{8}$, to synthesize equatorial tetrakis-cyclopropanated- $\mathrm{C}_{60}$ homo and hetero-derivatives, as well as hexakishetero-derivatives (all functionalized adducts are named as non-encapsulated compounds for clarity).

In general, the regiochemistry of exohedral poly-functionalization of fullerenes remains a standing problem. In order to gain control over these reactions, $\mathrm{C}_{60}$ was encapsulated within 1a. $(\mathrm{BArF})_{8}$, taking advantage of the complementary size and high affinity between the host and the guest. ${ }^{[15]}$ To test the strategy shown in Fig. 1c we first subjected $\mathrm{C}_{60} \subset \mathbf{1 a} \cdot(\mathrm{BArF})_{8}$ to standard conditions commonly used for Bingel-Hirsch reactions. Accordingly, diethyl bromomalonate (4 equiv.) and $\mathrm{NaH}$ (4 equiv.) were added into a solution of $\mathrm{C}_{60} \subset \mathbf{1 a} \cdot(\mathrm{BArF})_{8}$ in pure acetonitrile, and the resulting mixture was stirred during 2 hours. Electrospray ionization-high resolution mass spectrometry (ESI-HRMS) monitoring of the reaction crude revealed a stepwise formation of a single product corresponding to the encapsulated tetrakis-diethylmalonate- $\mathrm{C}_{60}$ adduct 2 (Fig. 1c, 2-3). ${ }^{1} \mathrm{H}$ NMR spectrum and HPLC chromatography showed only peaks corresponding to a single, regioisomerically pure, tetrakis-adduct in $>99.5 \%$ purity and $99 \%$ yield (Fig. 1-2). Further analysis by NMR and UV-vis unambiguously assigned the tetrakis-adduct 2 to a $\mathrm{D}_{2 \mathrm{~h}}$-symmetric e,e,e,eregioisomer (Fig. 2). Remarkably, this tetrakis-adduct 2 could be released from the nanocapsule either by exchange with pristine $\mathrm{C}_{60}$ or by disassembling the nanocapsule, which could be further reassembled and re-used. ${ }^{[15]}$

To evaluate the influence of nanocapsule 1a. $(\mathrm{BArF})_{8}$, we reproduced the Bingel-Hirsch reaction using free $\mathrm{C}_{60}$. Under these conditions, no reaction took place presumably due to the very poor solubility of $\mathrm{C}_{60}$ in acetonitrile. For this reason, we repeated this reaction in toluene where $\mathrm{C}_{60}$ is soluble. In toluene, from bis- to hexakis-adducts were produced even at short reaction times (30 min.), whereas up to $60 \%$ of starting $\mathrm{C}_{60}$ remained unreacted (Fig. S6). Altogether, these results suggested that the confinement of $\mathrm{C}_{60}$ in the nanocapsule completely modifies not only its reactivity in acetonitrile but also the reaction outcome, directing the nucleophilic attacks exclusively towards the equatorial $[6,6]$ bonds of the fullerene.

Slow diffusion of $\mathrm{Et}_{2} \mathrm{O}$ into a solution of $\mathbf{2} \subset \mathbf{1 b} \cdot(\mathrm{BArF})_{8}$ in $\mathrm{CH}_{2} \mathrm{Cl}_{2}$ containing $5 \%$ of $\mathrm{CH}_{3} \mathrm{CN}$ (1b- $(\mathrm{BArF})_{8}$ is the $\mathrm{Cu}$-based analog of the $\mathrm{Pd}$-based 1a. $\left.(\mathrm{BArF})_{8}\right)$, provided $\mathrm{X}$-ray quality single crystals suitable for diffraction analysis under synchrotron radiation. Single-crystal XRD analysis of $\mathbf{2} \subset \mathbf{1 b} \cdot(\mathrm{BArF})_{8}$ revealed that each diethylmalonate group of the docked tetrakis-adduct $\mathbf{2}$ was 
pointing towards the four apertures of nanocapsule $\mathbf{1 b} \cdot(\mathrm{BArF})_{8}($ Fig. 2). The short $\mathrm{Zn} \cdots \mathrm{Zn}$ distance $(13.17 \AA)$ indicated an important degree of adaptability of $\mathbf{1 b} \cdot(\mathrm{BArF})_{8}$ to maximize the porphyrinfullerene interactions. The fact that tetrakis-adduct 2 could be observed in the XRD analysis strongly suggested that rotation of the guest inside the nanocapsule was severely restricted. The latter was confirmed by Molecular Dynamics (MD) simulations of $\mathbf{2} \subset \mathbf{1 a} \cdot \mathrm{Cl}_{8}$, showing that motion of each diethylmalonate adduct is totally restricted within a single aperture of the nanocapsule due to their size (Fig. 3), although malonate groups do not stay fixed in the center of the open gates and they orient closer to the nanocapsule walls establishing persistent $\mathrm{C}-\mathrm{H} \cdots \pi$ interactions between alkyl ester and the aromatic rings of the nanocapsule. Indeed, the mean $\mathrm{Zn} \cdots \mathrm{Zn}$ distance in the MD (13.1 $\AA$ ) reproduced perfectly the one found by XRD, , and it is also in line with the distance measured in our previously reported DFT optimized $\mathrm{C}_{60} \subset \mathbf{1 a} \cdot \mathrm{Cl}_{8}$ structure. ${ }^{[15]}$

As expected, using other nucleophiles such as dimethyl bromomalonate, the $\mathrm{D}_{2 \mathrm{~h}}$-symmetric $e, e, e, e$-regioisomer of tetrakis-dimethylmalonate- $\mathrm{C}_{60}$ adduct $(3)$ was obtained in one step following the same procedure (Fig. 4). 
a.
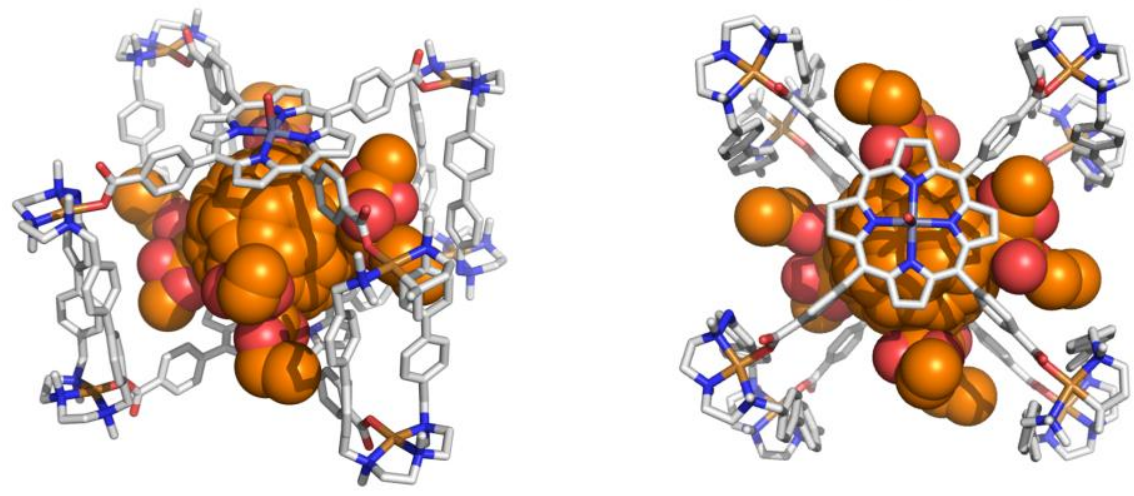

b.

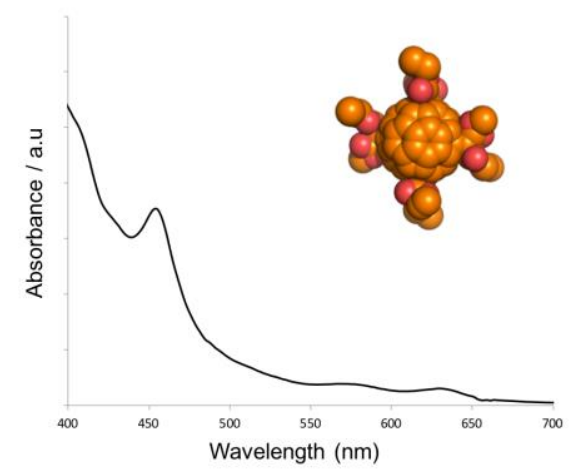

d.

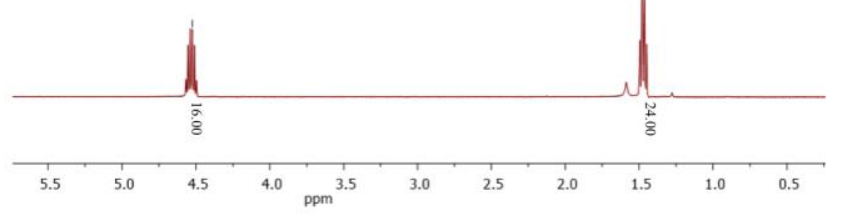

c.

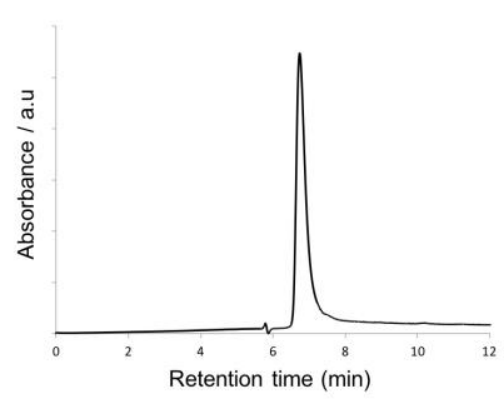

e.

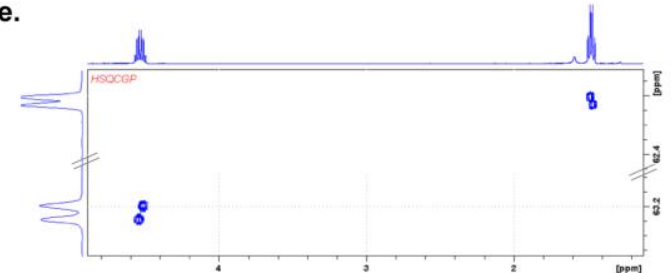

Fig. 2.Full characterization of $\boldsymbol{e}, \boldsymbol{e}, \boldsymbol{e}, \boldsymbol{e}$-tetrakis adduct (2). a) X-ray crystal structure of $\mathbf{2} \subset \mathbf{1 b} \cdot(\mathrm{BArF}){ }_{8}$ (side and apical view), depicting the tetrakis-adduct $\mathbf{2}$ docked in the nanocapsule $\mathbf{1 b} \cdot(\mathrm{BArF})_{8}$ (solvent, anions and $\mathrm{H}$ atoms are removed for clarity). $\mathbf{b}$ ) UV-vis, c) HPLC, d) ${ }^{1} \mathrm{H}-\mathrm{NMR}$ and e) 2D-SA-HSQC of isolated tetrakis-adduct 2. 
a.

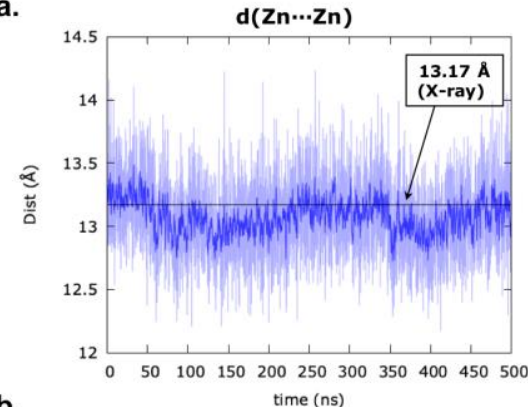

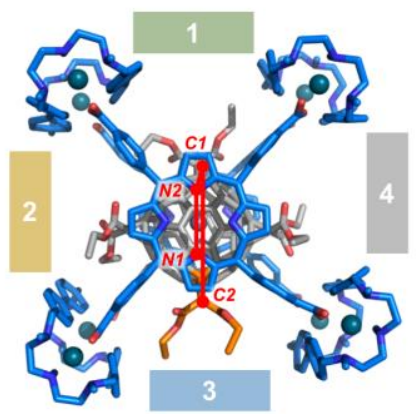

$\angle \mathrm{N} 1-\mathrm{N} 2-\mathrm{C} 1-\mathrm{C} 2$

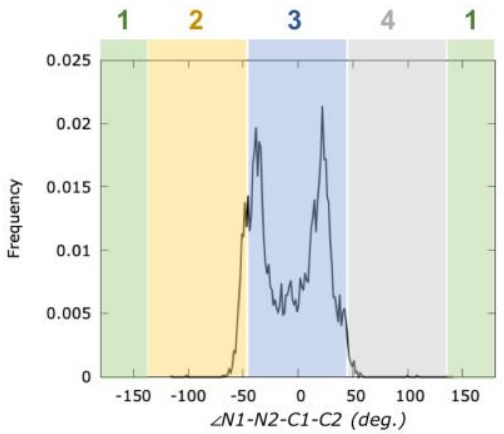

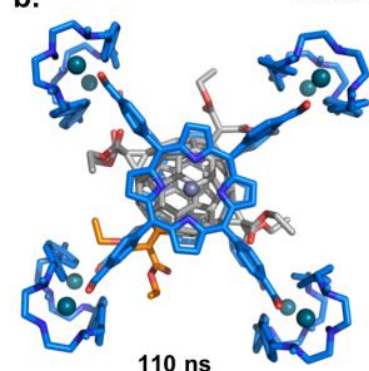

$110 \mathrm{~ns}$

$d(Z n \cdots Z n)=13.0 \AA$

$\angle \mathrm{N} 1-\mathrm{N} 2-\mathrm{C} 1-\mathrm{C} 2=-25.6^{\circ}$

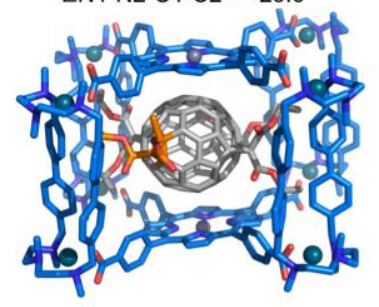

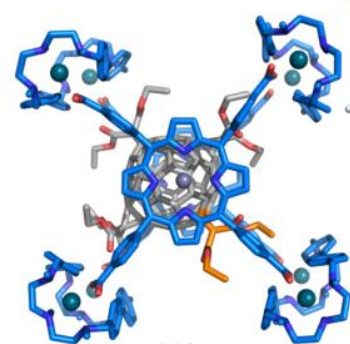

$314 \mathrm{~ns}$

$\mathrm{d}(\mathrm{Zn} \cdots \mathrm{Zn})=13.3 \AA$

$\angle \mathrm{N} 1-\mathrm{N} 2-\mathrm{C} 1-\mathrm{C} 2=36.4^{\circ}$

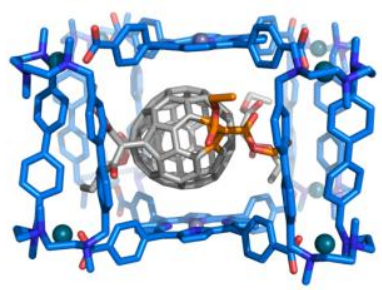

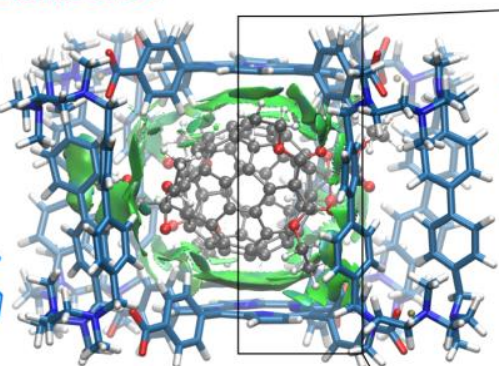

$162 \mathrm{~ns}$

$d(Z n \cdots Z n)=12.9 \AA$

$\angle \mathrm{N} 1-\mathrm{N} 2-\mathrm{C} 1-\mathrm{C} 2=18.6^{\circ}$

Persistent $\mathbf{C}-\boldsymbol{H} \cdots \pi$ interactions between ethyl ester groups and 1a stabilize the 2 $\subset 1$ a host-guest complex

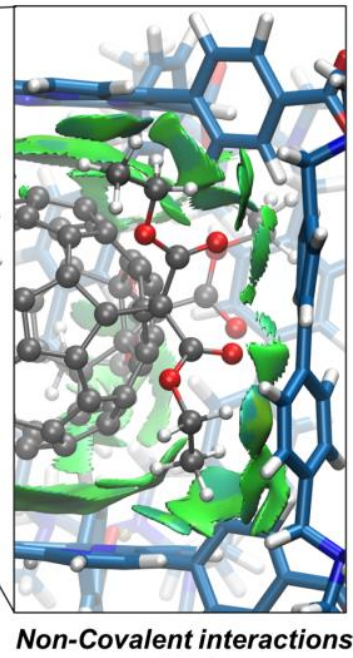

(NCI)

Fig. 3. a) Analysis of geometric features of $2 \subset \mathbf{1 a} \cdot(\mathrm{Cl})_{8}$ complex from $500 \mathrm{~ns}$ Molecular Dynamics (MD) simulations (distances are given in $\AA$ and dihedral angles in degrees). b) Snapshots of $\mathbf{2} \subset \mathbf{1 a} \cdot(\mathrm{Cl}) 8$ complex from MD trajectory showing different representative orientations of the encapsulated substrate 2. Non-covalent interaction analysis (NCI) revealed the appearance of persistent $\mathrm{C}-\mathrm{H} \cdots \pi$ interactions (stabilizing interactions shown as green surfaces) between ester alkyl and the aromatic rings of the nanocapsule. 
a
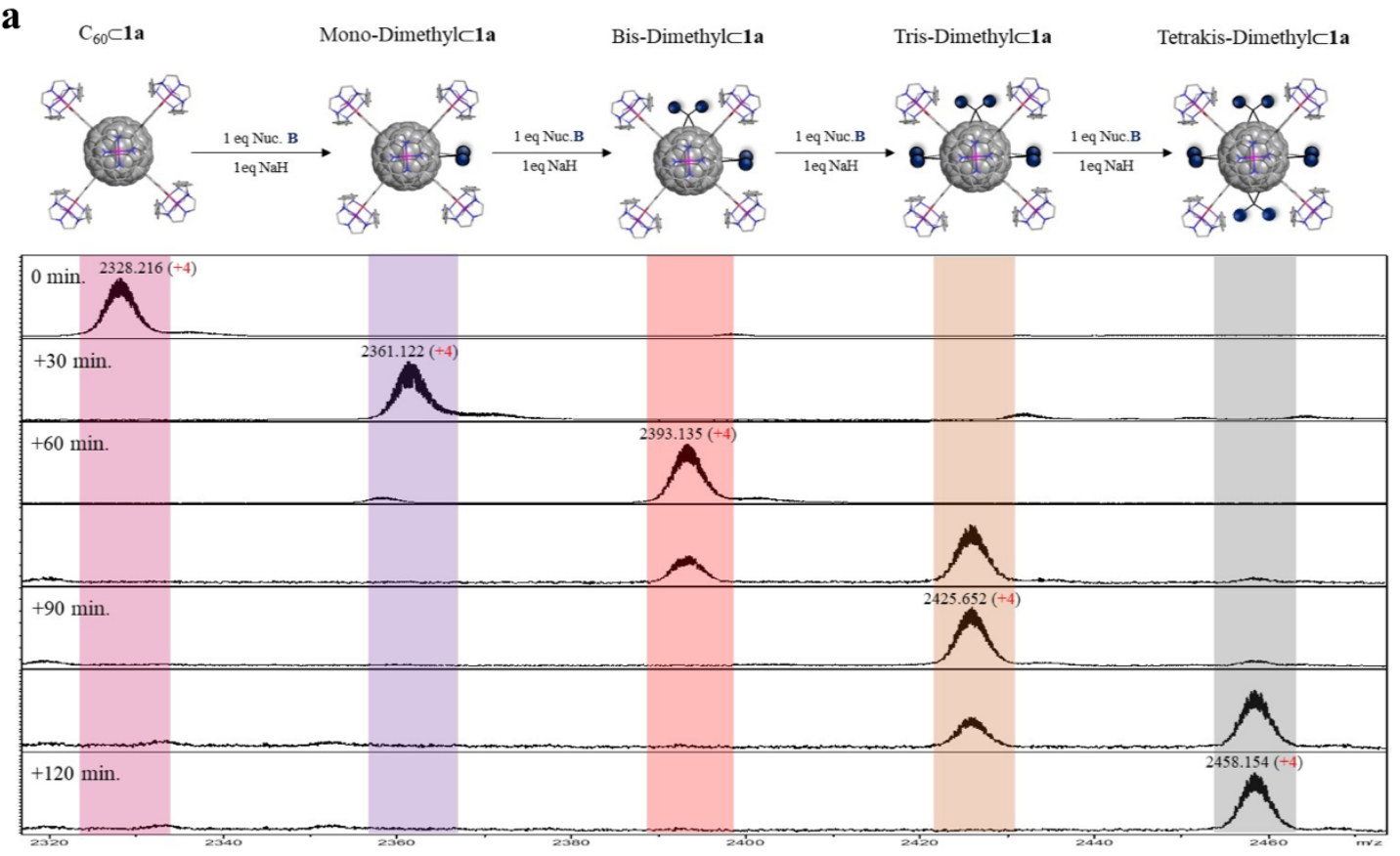

b

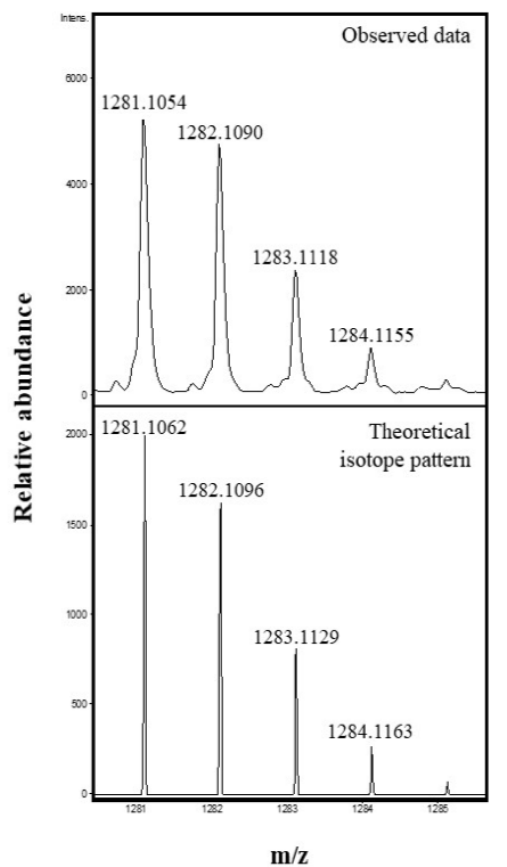

c
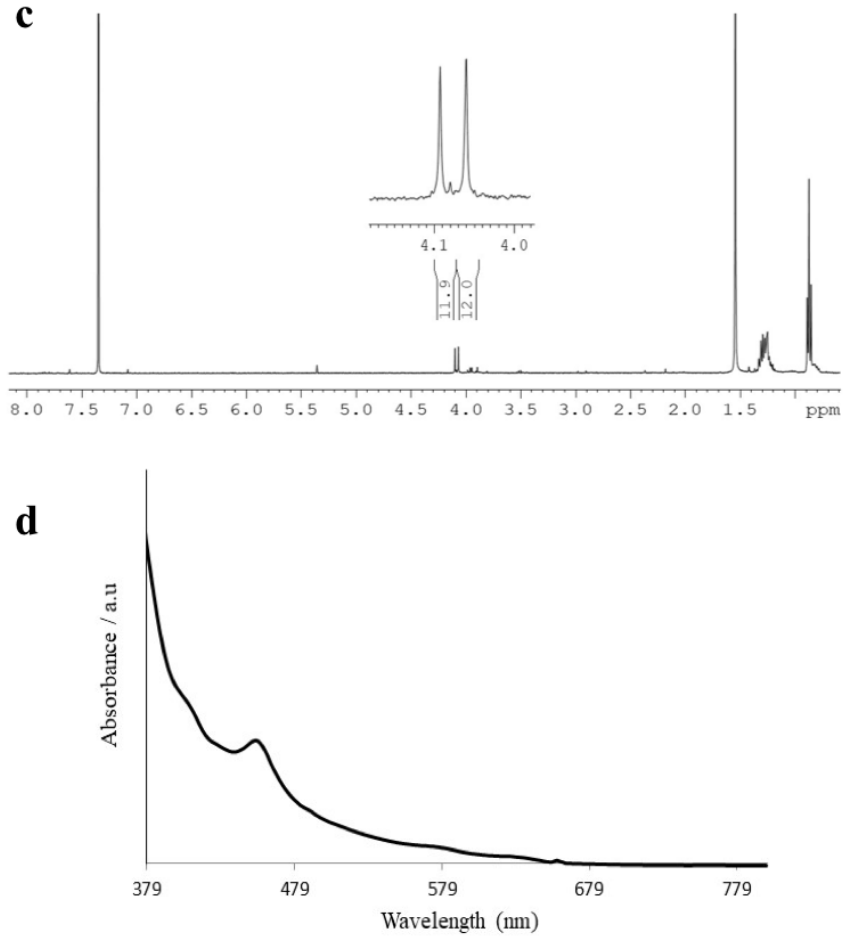

Fig. 4. Synthesis of equatorial e,e,e,e-tetrakis-dimethylmalonate- $\mathbf{C}_{60}$ (3). a, Mass spectrum monitoring the formation of $\mathbf{3} \subset \mathbf{1 a} \cdot(\mathrm{BArF})_{8}\left(\mathrm{ESI}-\mathrm{MS}\right.$, Positive ion mode, $\left.\mathrm{CH}_{3} \mathrm{CN}\right)$. b. Experimental and theoretical isotope pattern of high resolution ESI-MS of $\mathbf{3}\left(\mathrm{M}+\mathrm{Na}\right.$ ) (ESI-MS, Positive ion mode, $\mathrm{CH}_{2} \mathrm{Cl}_{2}: \mathrm{MeOH}$ ). $\mathbf{c},{ }^{1} \mathrm{H}-\mathrm{NMR}$ spectrum of adduct $3\left(500 \mathrm{MHz}, 298 \mathrm{~K}, \mathrm{CDCl}_{3}\right)$. d, UV-vis absorption spectrum of tetrakis-diethylmalonate- $\mathrm{C}_{60}$ adduct 3 (298 K, Toluene). 
It is known that functionalized $\mathrm{C}_{60}$ adducts are more prone to nucleophilic attacks than pristine $\mathrm{C}_{60}$. However, this over-reactivity was completely suppressed when $\mathrm{C}_{60}$ was encapsulated in 1a. $(\mathrm{BArF})_{8}$, observing a smooth stepwise formation of mono-, bis-, tris- and tetrakis-adducts (see Fig. 1 and 5). Following the herein reported supramolecular mask strategy, the single bis-adduct (4) was selectively produced by using sodium acetate as a weaker base and controlling the number of equivalents of nucleophile added (Fig. 5). 1D/2D NMR, HPLC and UV-vis analyses of released 4 indicated the exclusive formation of $\mathrm{C}_{\mathrm{s}}$-symmetric $e$,e-regioisomer, contrasting with the seven bis-regioisomers obtained in bulk solution (Fig. 5). No trace of trans-1 bis-adduct was detected either, which could be related to the enhanced reactivity in the equatorial bonds adjacent to the first addend. 


\section{Homo-adducts}
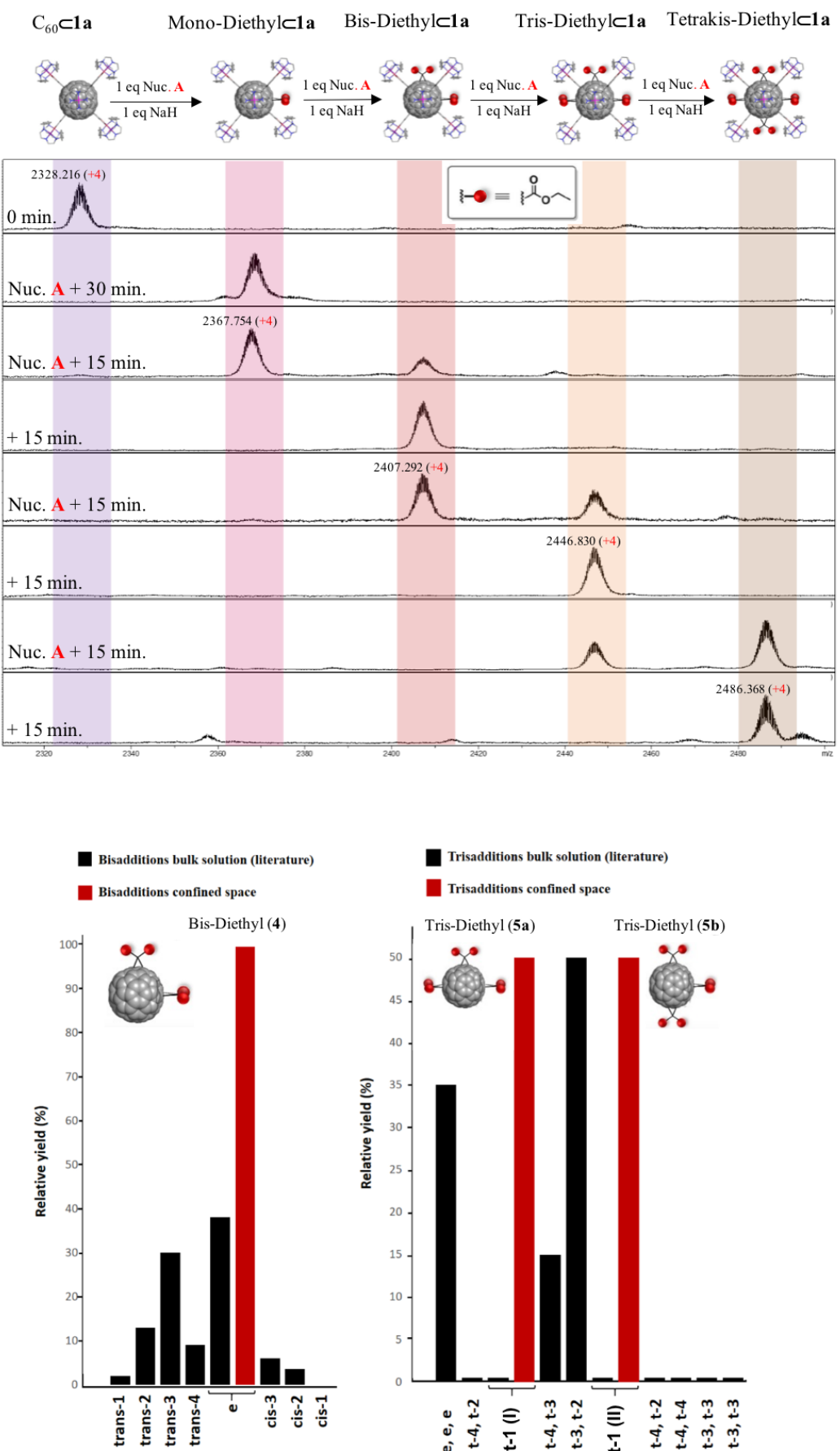

Trisadditions confined space

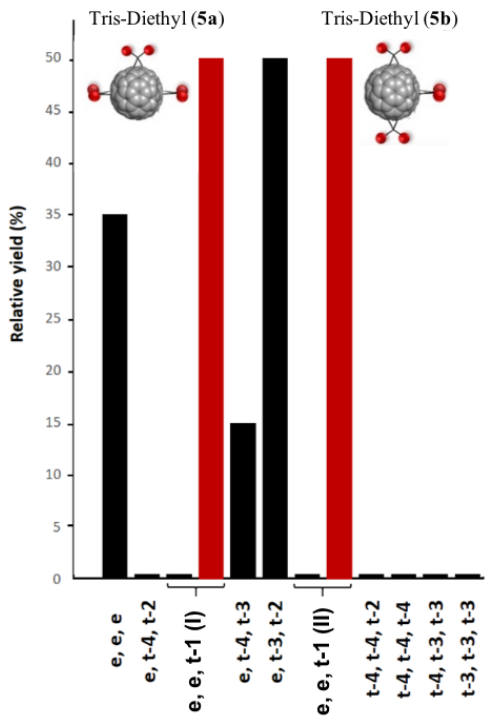

Fig. 5. ESI-HRMS spectra of stepwise formation of bis-, tris-, and tetrakis-equatorial adducts upon the sequential addition of nucleophile and base (top). Comparison between $\mathrm{C}_{60}$ regioisomers obtained in bulk solution and using the supramolecular confinement strategy for bis-adducts and tris-adducts (down) (Nuc. A = diethyl bromomalonate). 
Additionally, the equatorial tris-adduct was also attained (Fig. 1c), obtaining equimolar amounts of two non-chiral isomers $\mathbf{5 a}$ and $\mathbf{5} \mathbf{b}$, which corresponded to a $\mathrm{C}_{\mathrm{s}}$-symmetric e,e,t-1 (I) and $e, e, t-1$ (II), ${ }^{[33]}$ as ascertained by 1D/2D NMR (Fig. S2). Remarkably, both $e, e, t-1$ (I) and $e, e, t$ 1 (II) equatorial regioisomers have never been detected in bulk solution (Fig. 5). The steric constrains of the encapsulated bis-adduct 4 prevent free rotation as ascertained by MD simulations, exposing the two remaining equatorial sites to the nanocapsule apertures, being both equally suitable to further react forming tris-adducts $\mathbf{5 a}$ and $\mathbf{5 b}$.

Given the versatility of platform $\mathbf{1 a} \cdot(\mathrm{BArF})_{8}$, we pursued the development a synthetic route to produce equatorial hetero-tetrakis-adducts in a one-pot protocol. Noteworthy, it was required to use a single isomer as the precursor molecule to obtain a single tetrakis-regioisomer. The smooth and stepwise formation of cyclopropanated fullerene equatorial adducts when $\mathrm{C}_{60}$ is confined in 1a. $(\mathrm{BArF})_{8}$, allowed in operando synthesis of the $e, e$-dimethyl-bis-adduct. The subsequent addition of 2 equiv. of diethyl bromomalonate and 2 equiv. of sodium acetate on the reaction crude afforded the fully equatorial hetero- $\mathrm{D}_{2 \mathrm{~h}}$-tetrakis-adduct $e, e$-bis-diethyl-e,e-bis-dimethylmalonate$\mathrm{C}_{60}$ (6) (Fig. 6), as a single isomer in high purity and excellent yield (97\% yield). The same synthetic strategy was followed changing the nucleophile once mono-diethylmalonate- $\mathrm{C}_{60}$ adduct was obtained. The fully equatorial hetero-tetrakis-adduct e,e,t-1(I)-tris-dimethyl-e-monodiethylmalonate- $\mathrm{C}_{60}(\mathbf{7 a})$ was formed upon the addition of 3 equiv. of dimethyl bromomalonate and 3 equiv. of sodium hydride on the reaction crude (Fig. 1c). UV-vis spectrum indicated that a fully equatorial compound was obtained. However, careful 1D/2D NMR analysis showed that the isomer $7 \mathbf{b}\left(e, e, t-1(I I)\right.$-tris-dimethyl-e-mono-diethylmalonate- $\left.\mathrm{C}_{60}\right)$ was also present in a minor amount (2.5:1 7a:7b) (Fig. S4). To better understand the parameters governing this preferential formation, the same synthetic strategy was followed using with mono-dimethylmalonate- $\mathrm{C}_{60}$ adduct as precursor and adding 3 equiv. of diethyl bromomalonate. Again, a mixture of heterotetrakis-adduct $e, e, t-1(I)$-tris-diethyl-e-mono-dimethylmalonate- $\mathrm{C}_{60}$ (8a) and e,e,t-1(II)-trisdiethyl-e-mono-dimethylmalonate- $\mathrm{C}_{60}(\mathbf{8 b})$ was obtained in a similar ratio (2.8:1 8a:8b) (Fig 7). MD simulations showed totally restricted gate-to-gate motion of bis-adducts, as opposite to the mono-adducts, which can also spin around the cyclopropane axis (Fig. 8 and Fig. S8). Taken together, these results suggested that a preferential orientational attack of the second nucleophile fixed the fullerene molecule to produce 7a and $\mathbf{8 a}$ in higher ratios, and that the preference of this 
second addition might be related to the non-covalent interactions between alkyl ester and the aromatic rings of the nanocapsule observed by MD (Fig. S9).

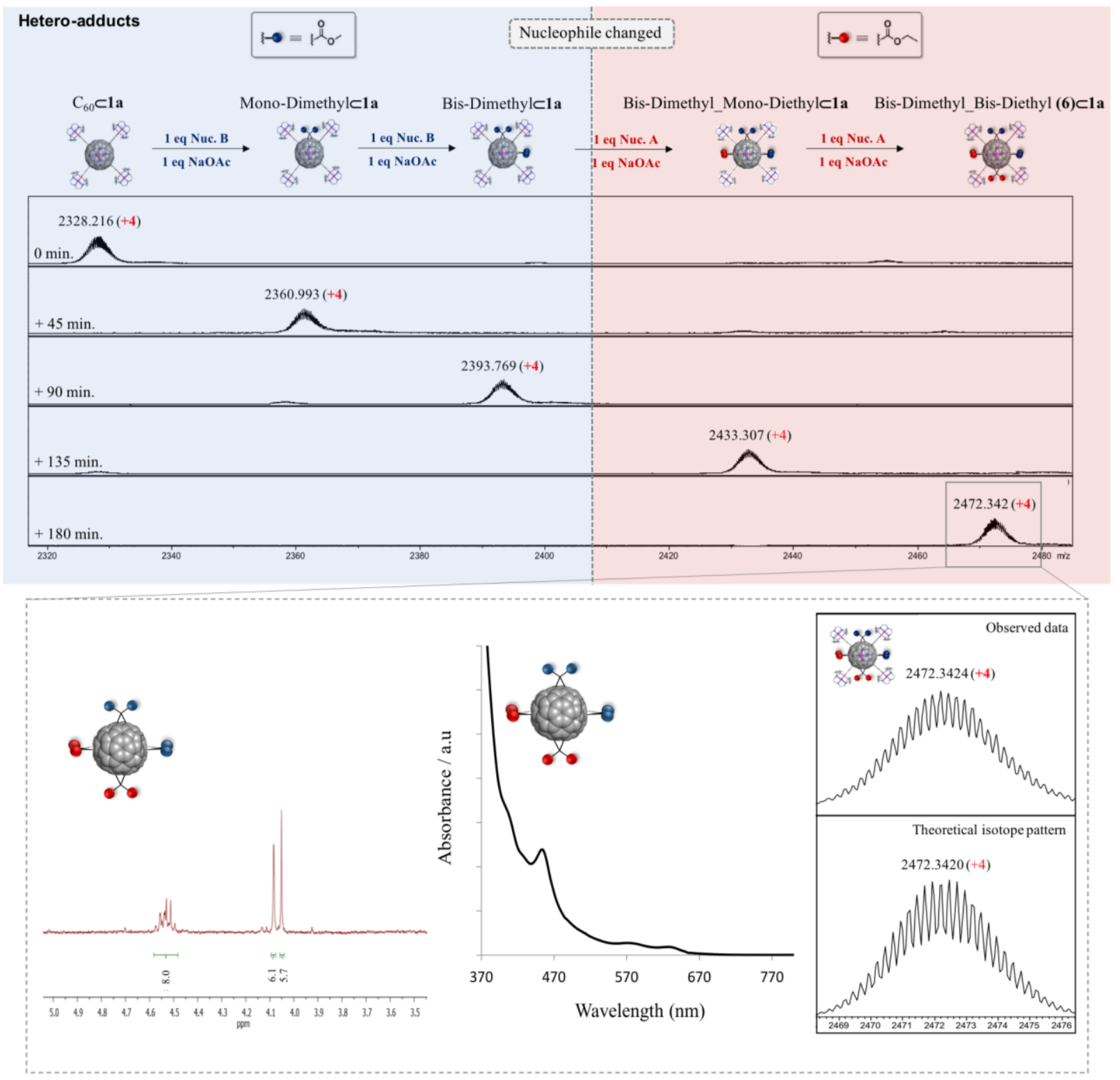

Fig. 6. Stepwise synthesis of hetero-tetrakis-adduct $e, e$-bis-diethyl-e,e-bis-dimethylmalonate-C 60 (Nuc. B = dimethyl bromomalonate). Inset shows the ${ }^{1} \mathrm{H}$ NMR, UV-Vis and MS (isotopic pattern for $\mathrm{z}=+4$ ) characterization for 6. 
a

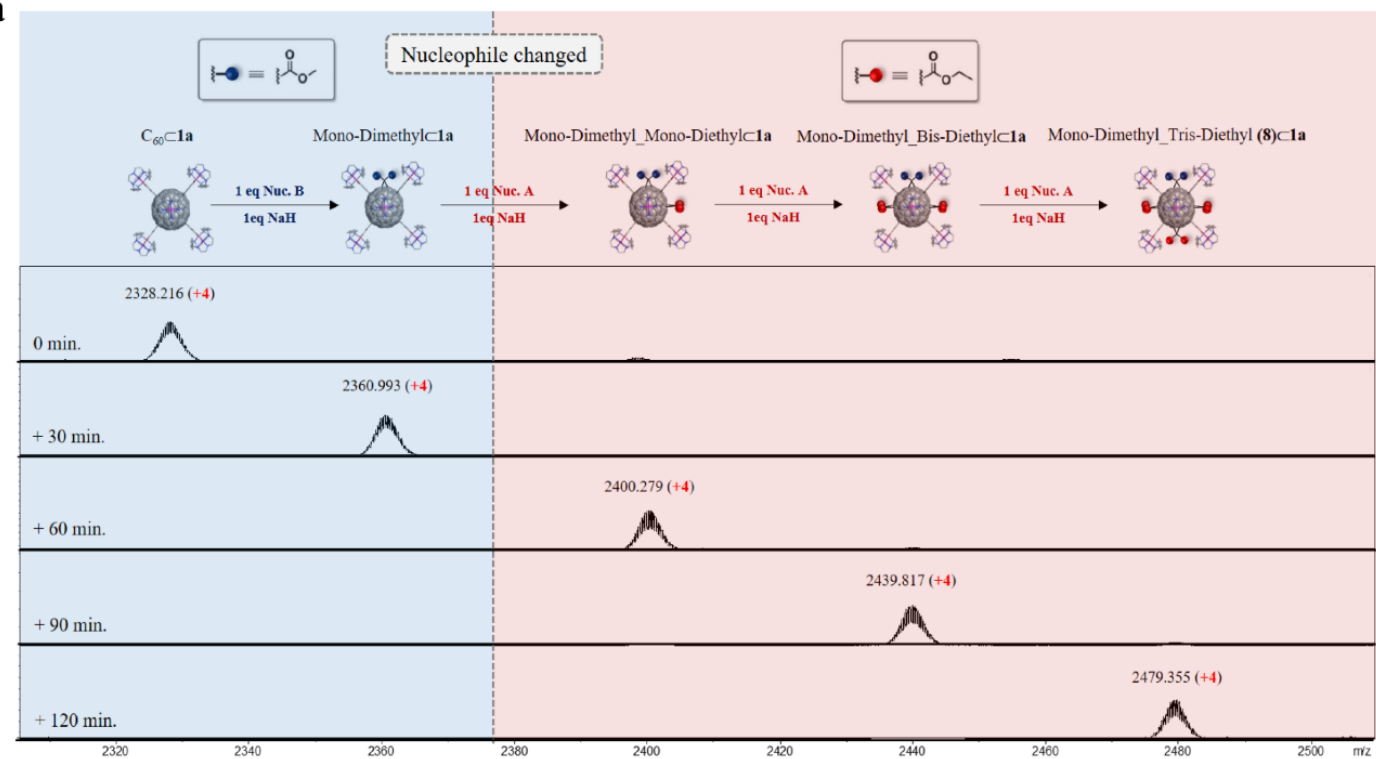

b

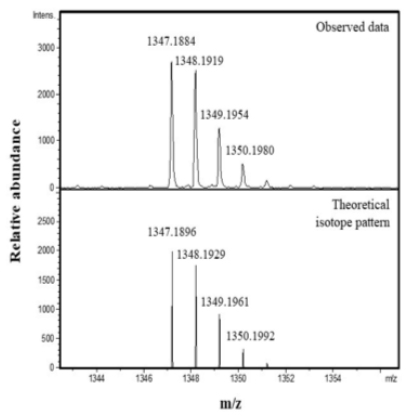

c

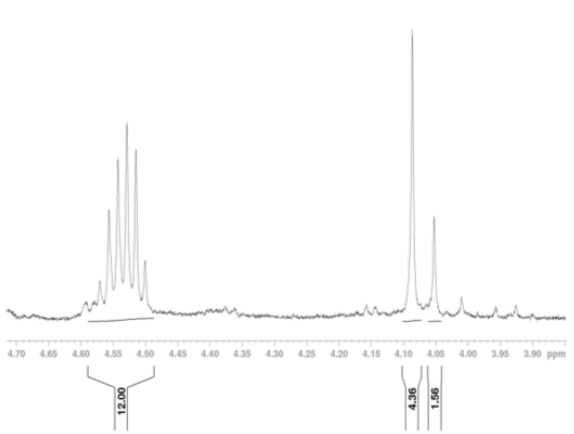

f

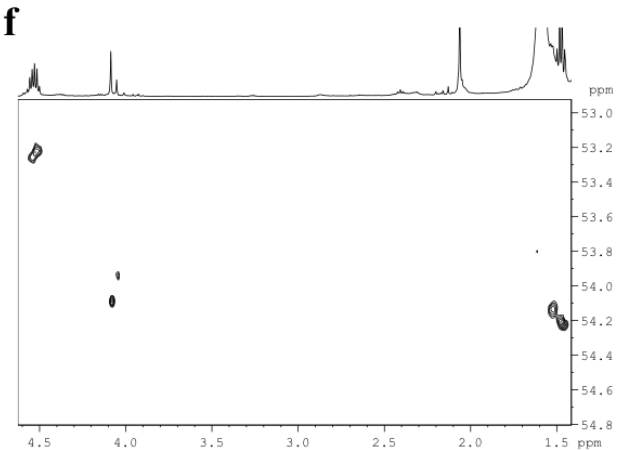

d

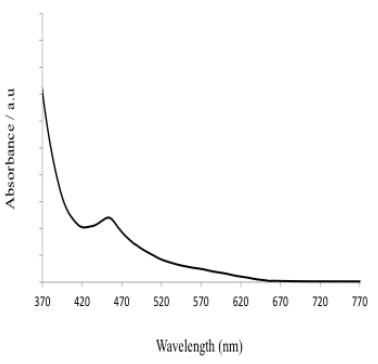

e

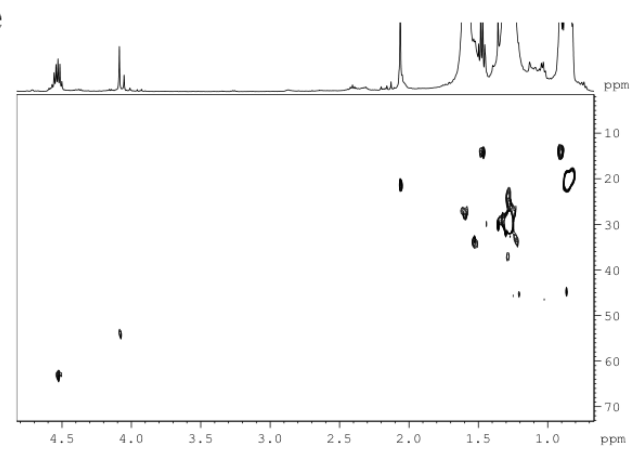



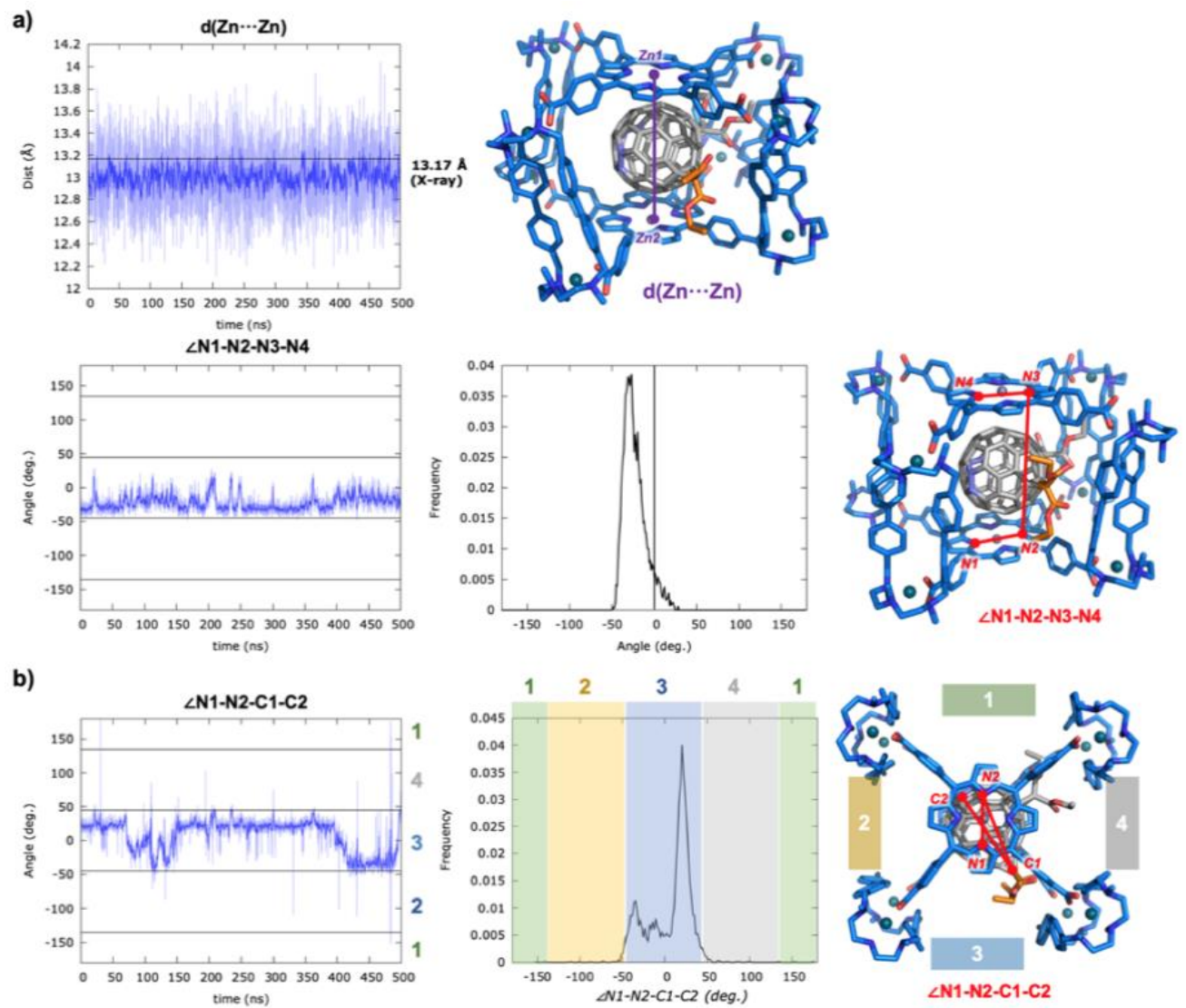

c)
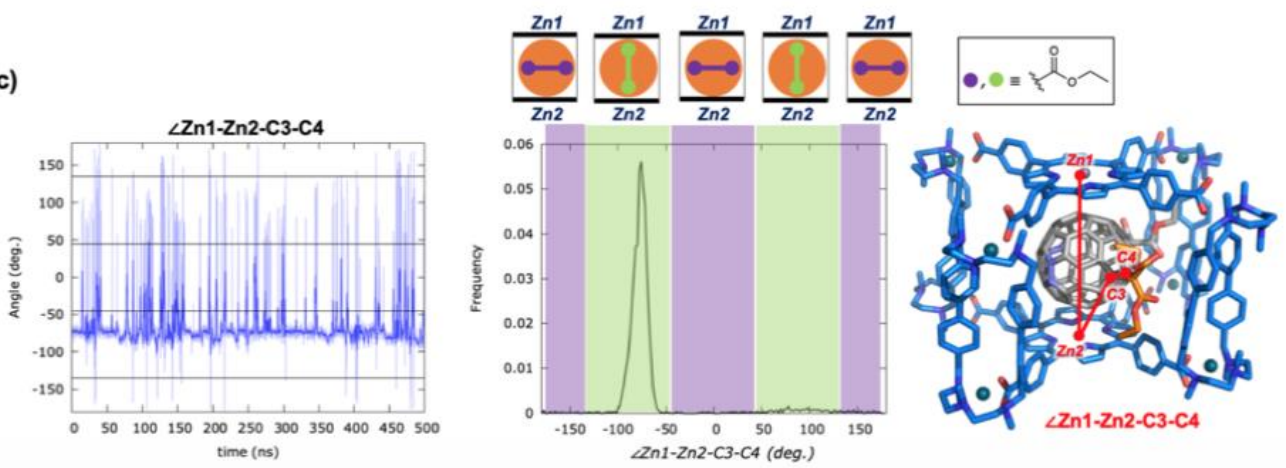

Fig. 8. Conformational analysis of $4 \subset 1 \mathrm{a} \cdot(\mathrm{Cl})_{8}$ (bis-e,e-adduct) host-guest complex from $500 \mathrm{~ns}$ of Molecular Dynamics (MD) trajectories in explicit $\mathbf{C H}_{3} \mathbf{C N}$ solvent. a, Zn $\cdots$ Zn distance and $\angle \mathrm{N} 1-\mathrm{N} 2-\mathrm{N} 3-\mathrm{N} 4$ dihedral angle measured along the MD trajectory. $\angle \mathrm{N} 1-\mathrm{N} 2-\mathrm{N} 3-\mathrm{N} 4$ dihedral angle describes the torsion of the nanocapsule: the larger deviation from $\angle \mathrm{N} 1-\mathrm{N} 2-\mathrm{N} 3-\mathrm{N} 4=0$ angle, the more twisted the capsule. Most visited angle values are shown in the histogram plot (frequency vs. angle value). b, $\angle \mathrm{N} 1-\mathrm{N} 2-\mathrm{C} 1-\mathrm{C} 2$ dihedral angle measured along the MD trajectory. $\angle \mathrm{N} 1-\mathrm{N} 2-\mathrm{C} 1-\mathrm{C} 2$ angle describes the relative rotation of the encapsulated fullerene with respect to the capsule, and most visited angle values are shown in the histogram plot (frequency vs. angle value). Different capsule windows (labelled as 1, 2, 3, and 4 in the figure) correspond to different ranges of explored angle values. c, $\angle \mathrm{Zn} 1-\mathrm{Zn} 2-\mathrm{C} 3-\mathrm{C} 4$ dihedral angle measured along the MD trajectory. $\angle \mathrm{Zn} 1-\mathrm{Zn} 2-\mathrm{C} 3-\mathrm{C} 4$ angle describes the relative orientation of the cyclopropane ring with respect to the capsule. $\angle \mathrm{Zn} 1-\mathrm{Zn} 2-\mathrm{C} 3-\mathrm{C} 4$ values around $0^{\circ}$ and $\pm 180^{\circ}$ indicate that the cyclopropane ring stays perpendicular to the porphyrins, while values around $\pm 90^{\circ}$ indicate that the cyclopropane ring stays in a parallel conformation with respect to the porphyrins. Distances and angles are given in Angstroms (A) and degrees $\left({ }^{\circ}\right)$, respectively 
In a further twist in the preparation of hetero-polyadducts, we discovered that by adding more equivalents ( 3 equiv) of a different malonate (i.e. dimethylbromomalonate) after the synthesis of $e, e, e, e$-tetrakis-diethylmalonate- $\mathrm{C}_{60}$ adduct (2) inside the nanocapsule, a pure single isomer of the $T h$-hexakis-hetero-adduct, namely e,e,e,e-tetrakis-diethylmalonate- $t$ - $1, t$ - 1 -bis-diethylmalonate$\mathrm{C}_{60}$ (9), was obtained (Fig. 1c and Fig. 9). ${ }^{[10]}$ Given the multiple options described in Fig. 1c, the present supramolecular mask strategy can be potentially used to obtain all possible combinations of $T h$-hexakis heteroadducts with two different addends ([6:0], [5:1], type I [1:5],type I [4:2], type II [4:2], type I [2:4], type II [2:4] and type II [3:3]), with the exception of type I [3:3]). ${ }^{[10,18]}$ Notably, diffusion coefficients (DOSY-NMR) of bis-, tris-, tetrakis- and hexakis adducts were in agreement with the increasing bulkiness of the molecules (Fig. S4). 
a

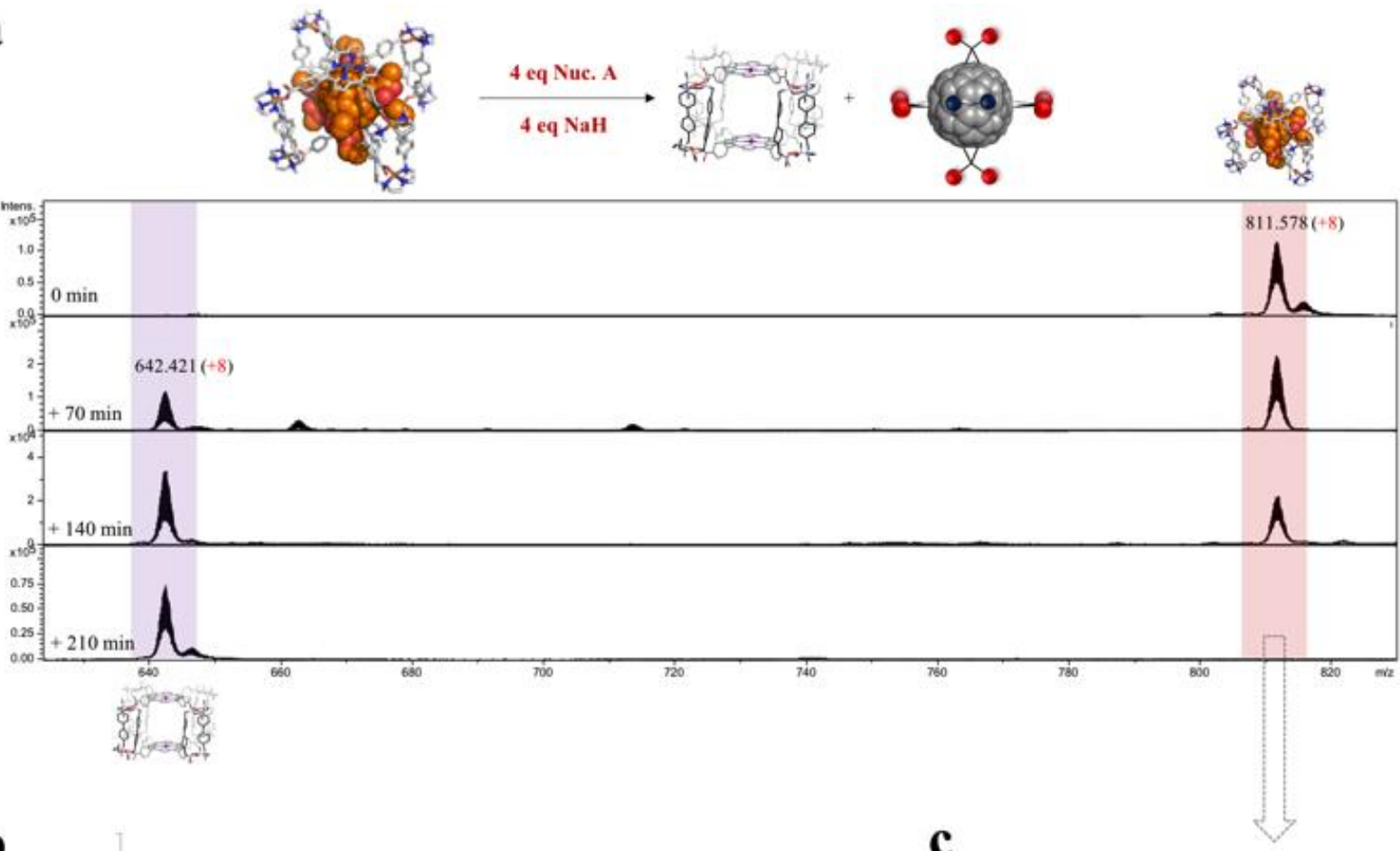

b
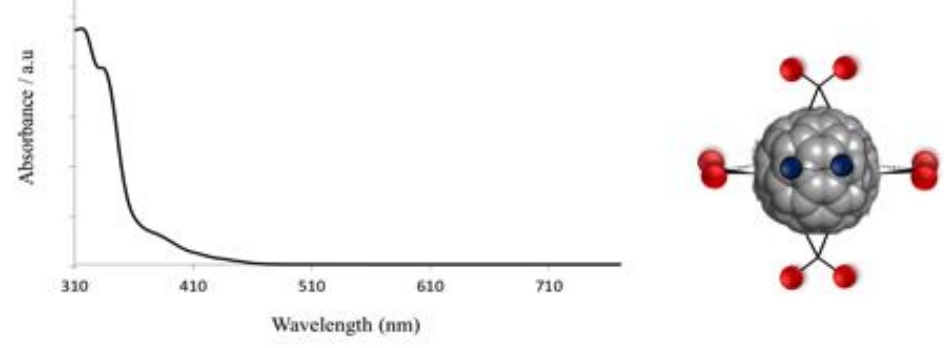

d
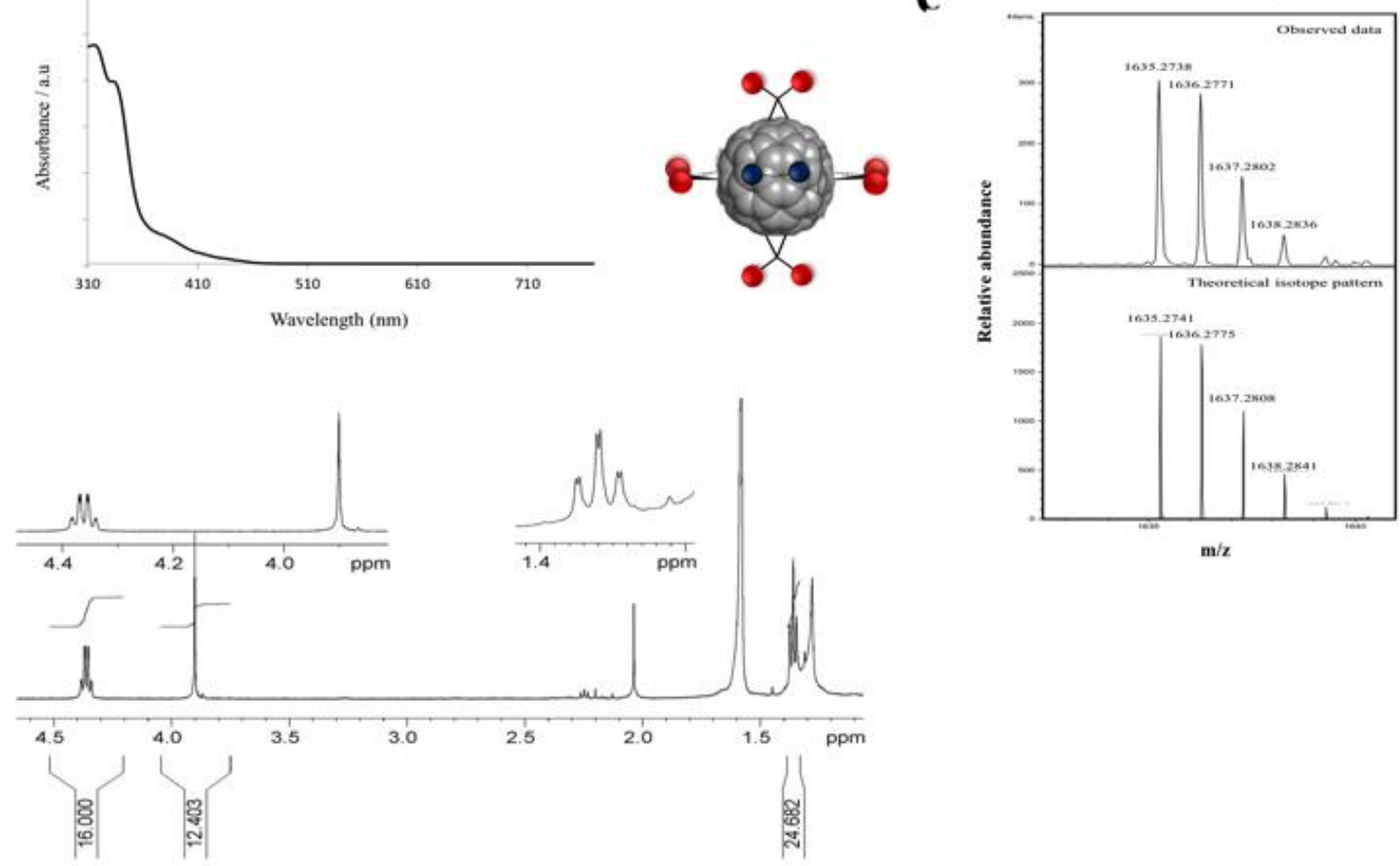

Fig. 9. Synthesis of $T h$-hexakis-hetero-adduct $e, e, e, e$-tetrakis-diethylmalonate-t-1,t-1-bis-diethylmalonate- $\mathrm{C}_{60}$ (9). a, HRMS monitoring for the formation of $\mathbf{9}$ from $\mathbf{2} \subset \mathbf{1 a} \cdot(\mathrm{BArF})_{8}$. Adduct $\mathbf{2}$ was released from $\mathbf{1 a} \cdot(\mathrm{BArF})_{8}$ upon the addition of Nuc. B, forming adduct $\mathbf{9}$ outside the capsule thus observing the formation of empty cage. (Nuc. B: Dimethyl bromomalonate). b, UV-vis absorption spectrum of adduct $\mathbf{9}$ (298 K, Toluene). c. ESI-MS of 9, positive ion mode, $\mathrm{CH}_{3} \mathrm{CN}$. d. ${ }^{1} \mathrm{H}-\mathrm{NMR}$ spectrum of adduct $9\left(500 \mathrm{MHz}, 298 \mathrm{~K}, \mathrm{CDCl}_{3}\right)$. 
Finally, the supramolecular mask concept was upgraded to a synthetically useful catalytic methodology by implementing a reversible phase transfer protocol. Firstly, we sough confirmation that the nanocapsule (with and without cargo) could be cleanly be transferred by anion metathesis from the organic phase (toluene: $\mathrm{CH}_{2} \mathrm{Cl}_{2}$ 1:1) to the aqueous phase $\left(\mathrm{H}_{2} \mathrm{O}\right.$ :DMSO 9:1) after the addition of 5 equiv. of $\left(n \mathrm{Bu}_{4} \mathrm{~N}\right)_{2}\left(\mathrm{SO}_{4}\right)$, and vice versa after the addition of 10 equiv. of $\mathrm{NaBArF}$ (Fig. 10a). Once this was ensured, the catalytic system was set up dissolving 1 equiv. of $\mathbf{1 a} \cdot(\mathrm{BArF})_{8}$ and 20 equiv. of $\mathrm{C}_{60}$ in the organic layer. Then, by the addition of 5 equiv. of $\left(n \mathrm{Bu}_{4} \mathrm{~N}\right)_{2}\left(\mathrm{SO}_{4}\right)$, the cage and the cargo were transferred to the aqueous layer $\left(\mathrm{C}_{60} \subset \mathbf{1 a} \cdot\left(\mathrm{SO}_{4}\right)_{4}\right)$, triggering simultaneously the Bingel-Hirsch reaction (diethyl bromomalonate as nucleophile and $\mathrm{NaOAc}$ as base). This reaction required 24 hours due to the low solubility of diethyl bromomalonate in aqueous phase (10\% DMSO was added to enhance its solubility while maintaining the double phase). After completion, addition of 10 equiv. of NaBArF brought the encapsulated tetrakisadduct (i.e. $\mathbf{2} \subset \mathbf{1 a} \cdot(\mathrm{BArF})_{8}$ ) into the organic phase, which contained excess of $\mathrm{C}_{60}$ (19 equiv). In this situation, simple stirring for 30 minutes allowed the ejection of 2 (e,e,e,e-tetrakisdiethylmalonate- $\mathrm{C}_{60}$ ) into the organic phase by encapsulation of pristine $\mathrm{C}_{60}$, which has a $\sim 20$-fold higher affinity than the tetrakis-adduct $2\left(K_{\mathrm{a}}(2)=1.03 \cdot 10^{6} \mathrm{M}^{-1} ; K_{\mathrm{a}}\left(\mathrm{C}_{60}\right)=2.8 \cdot 10^{7} \mathrm{M}^{-1}\right)($ Fig. 10b). The excess of $\mathrm{C}_{60}$ was used to ensure a rapid exchange with 2 . Further addition of 5 equiv. of $\left(n \mathrm{Bu}_{4} \mathrm{~N}\right)_{2}\left(\mathrm{SO}_{4}\right)$ started the second cycle of the protocol. Repetition of 10 complete cycles afforded quantitative formation of $\mathbf{2}$, which was dried, separated from the remaining excess of $\mathrm{C}_{60}$ by flash chromatography and analyzed by HLPC to confirm its purity ( $>97 \%$ with respect to consumed $\mathrm{C}_{60}$, 10 TON, 9.7 equiv., $10.2 \mathrm{mg}$ ). Alternatively, the excess of $\mathrm{C}_{60}$ can be removed by the addition of precise amount of $\mathbf{1 a} \cdot(\mathrm{BArF})_{8}$ to exclusively form $\mathrm{C}_{60} \subset \mathbf{1 a} \cdot(\mathrm{BArF})_{8}$ owing to its higher affinity. The process was catalytic in nanocapsule $\mathbf{1 a} \cdot(\mathrm{BArF})_{8}$, no decomposition of the supramolecular host was detected after 10 cycles and its efficiency was kept quantitative provided $\mathrm{C}_{60}$ was found in excess with respect to the tetrakis-adduct. Moreover, since the Bingel-Hirsch reaction is confined at the aqueous phase, the tetrakis adduct can be accumulated in the organic phase with any sign of overreaction, thus avoiding one of main drawbacks in fullerene functionalization. The protocol meets the fullerene-derivative scale (tens of milligram) requirements for perovskite solarcell device preparation ${ }^{[1]}$ and might be easily scalable. Collectively, the present supramolecular one-pot mask protocol allows potential access to a plethora of new single regioisomer poly- 
functionalized $\mathrm{C}_{60}$-based compounds, which are candidates for testing in solar cell devices and other technological and biomedicinal applications.

a.

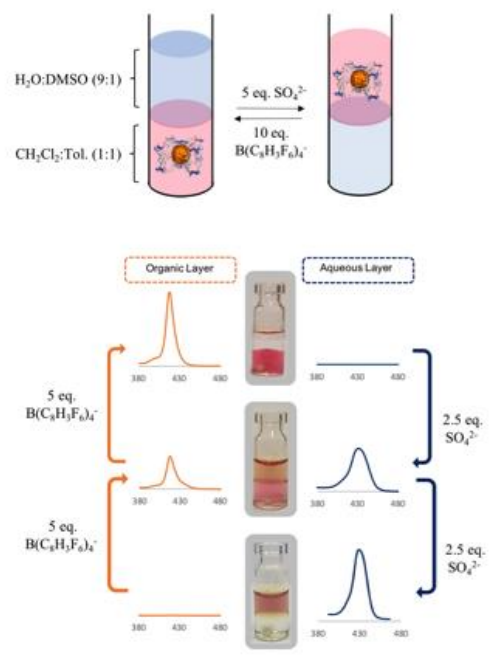

b. $2 c_{w} \quad$ Anion metathesis triggers Bingel-Hirsch reaction in aqueous phase

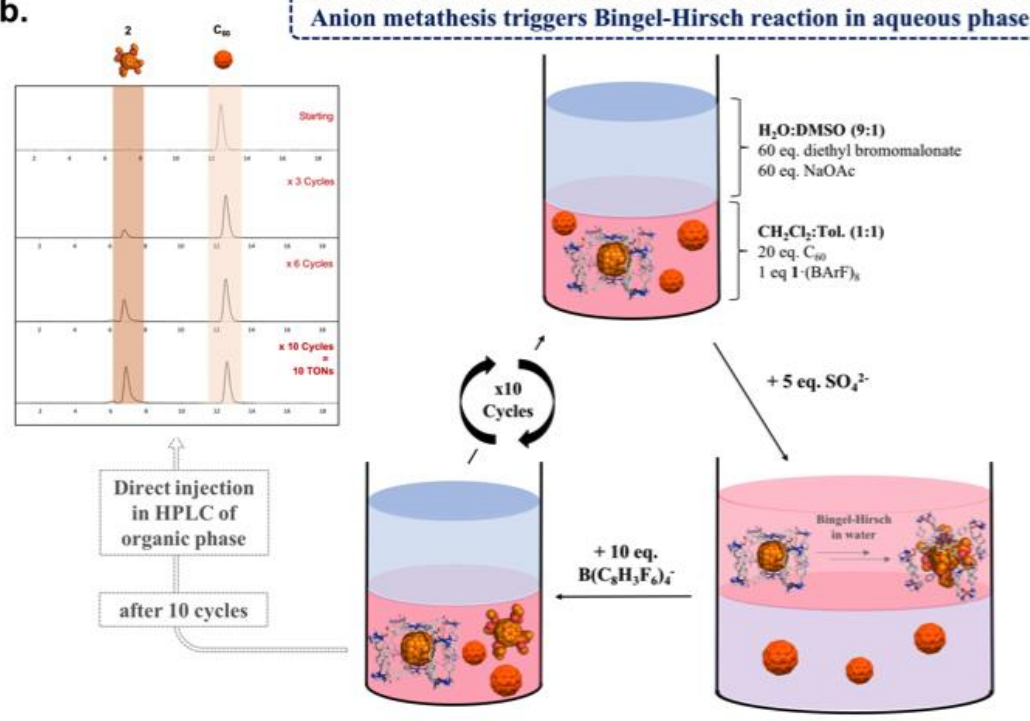

c.
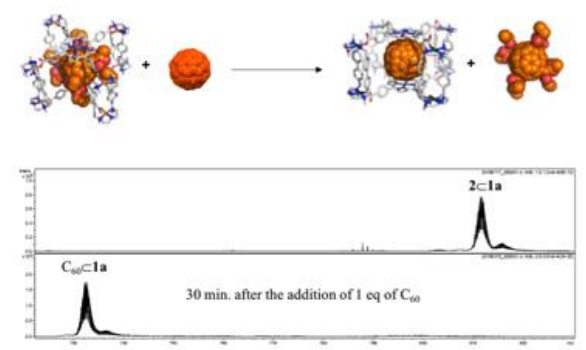

d.
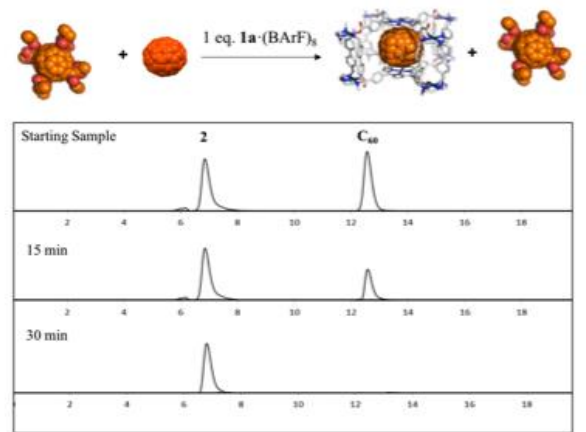

Fig. 10. Biphasic catalytic protocol for the synthesis of the equatorial tetrakis-adduct 2. a) Reversible phase transfer of $\mathrm{C}_{60} \subset \mathbf{1 a}$ from the organic to the aqueous phase and viceversa. b) Design of biphasic methodology for the synthesis of equatorial tetrakis-adduct 2 using $10 \mathrm{~mol} \%$ catalytic loadings of nanocapsule 1a.(BArF) 8 (up to $10 \mathrm{TONs}$ with no signs of decomposition of 1a). c) Control ESI-MS experiment for the exchange of encapsulated 2 by equimolar amount of $\mathrm{C}_{60}$ after 30 minutes. d) Control experiment showing the higher affinity of nanocapsule $\mathbf{1 a} \cdot(\mathrm{BArF})_{8}$ for $\mathrm{C}_{60}$ with equimolar amounts of $\mathbf{2}$ and $\mathrm{C}_{60}$.

In conclusion, a tetragonal prismatic supramolecular nanocapsule is used as a supramolecular mask to exert full control on the reactivity and the equatorial regioselectivity of Bingel-Hirsch cyclopropanation reactions of confined $\mathrm{C}_{60}$ fullerene. The four cross-shaped open windows featured by the supramolecular mask are key to gain full control on the functionalization of encapsulated $\mathrm{C}_{60}$ in terms of exclusive equatorial regioselectivity and stepwise formation of the 
bis-, tris- and tetrakis- $\mathrm{C}_{60}$ homo-adducts. Moreover, isomer-pure equatorial hetero-tetrakisadducts or hetero-Th-hexakis-adducts are synthesized at will in one-pot synthesis for the first time. To upgrade this strategy into a catalytic regime, a synthetically useful biphasic protocol is designed to achieve selective and quantitative formation of exclusively tetrakis-adducts, by using anion metathesis and catalytic amounts of nanocapsule. This work provides a synthetically valuable path to produce unprecedented pure-isomer poly-functionalized $\mathrm{C}_{60}$-based compounds, which are candidates for testing in solar cell devices and for biomedical applications. 
Supplementary Information is available in the online version of the paper.

\section{Acknowledgments}

This work was supported by grants from MINECO-Spain (CTQ2016-77989-P to X.R., MAT201565354-C2-1-R to D.M. and I.I., a JdC contract IJCI-2017-33411 to M.G.-B.), Generalitat de Catalunya (2017SGR264 and a PhD grant to C.F.-E.) and the Severo Ochoa Center of Excellence Program (ICN2, Grant SEV-2017-0706). X.R. and M.C. also thank ICREA-Acadèmia awards.

\section{Author contributions}

C.F.-E., C.G.-S. and M.P. performed all synthetic experiments and isolated all products. M.G.-B performed all Molecular Dynamics simulations and computational modeling analyses. L.G. provided technical assistance on HRMS studies. T.P. performed all NMR characterization analyses. J.J., I.I. and D.M. technically assisted, performed and solved the XRD structure of $\mathbf{2} \subset \mathbf{1 b} \cdot(\mathrm{BArF})_{8}$ at ALBA synchrotron. M.C and X.R. wrote the manuscript. X.R. directed the project.

\section{Author Information}

X-ray crystallographic data for for $\mathbf{2} \square \mathbf{1 b}$. $(\mathrm{BArF})_{8}$ has been deposited in the Cambridge Crystallographic Data Centre database (http://www.ccdc.cam.ac.uk/) under code CCDC 1913118. Reprints and permissions information is available at www.nature.com/reprints. The authors declare no competing financial interests. Readers are welcome to comment on the online version of the paper. Correspondence and requests for materials should be addressed to X.R. (xavi.ribas@udg.edu).

\section{Data and materials availability}

All additional data are in the supplementary materials.

\section{References}


[1] F. Zhang, W. Shi, J. Luo, N. Pellet, C. Yi, X. Li, X. Zhao, T. J. S. Dennis, X. Li, S. Wang, Y. Xiao, S. M. Zakeeruddin, D. Bi and M. Grätzel, (2017), Isomer-Pure Bis-PCBM-Assisted Crystal Engineering of Perovskite Solar Cells Showing Excellent Efficiency and Stability, Adv. Mater. 29, 1606806.

[2] M. Lenes, G.-J. A. H. Wetzelaer, F. B. Kooistra, S. C. Veenstra, J. C. Hummelen and P. W. M. Blom, (2008), Fullerene Bisadducts for Enhanced Open-Circuit Voltages and Efficiencies in Polymer Solar Cells, Adv. Mater. 20, 2116-2119.

[3] T. Umeyama and H. Imahori, (2019), Isomer Effects of Fullerene Derivatives on Organic Photovoltaics and Perovskite Solar Cells, Acc. Chem. Res.

[4] T. Umeyama, K. Igarashi, D. Sakamaki, S. Seki and H. Imahori, (2018), Unique cohesive nature of the $\beta 1$-isomer of [70]PCBM fullerene on structures and photovoltaic performances of bulk heterojunction films with PffBT4T-2OD polymers, Chem. Commun. 54, 405-408.

[5] T. Umeyama, T. Miyata, A. C. Jakowetz, S. Shibata, K. Kurotobi, T. Higashino, T.

Koganezawa, M. Tsujimoto, S. Gélinas, W. Matsuda, S. Seki, R. H. Friend and H. Imahori, (2017), Regioisomer effects of [70]fullerene mono-adduct acceptors in bulk heterojunction polymer solar cells, Chem. Sci. 8, 181-188.

[6] T. Gatti, E. Menna, M. Meneghetti, M. Maggini, A. Petrozza and F. Lamberti, (2017), The Renaissance of fullerenes with perovskite solar cells, Nano Energy 41, 84-100.

[7] E. Castro, J. Murillo, O. Fernandez-Delgado and L. Echegoyen, (2018), Progress in fullerenebased hybrid perovskite solar cells, J. Mater. Chem. C 6, 2635-2651.

[8] K. L. Maxouti and A. Hirsch, (2018), Sequential Tether-Directed Synthesis of PentakisAdducts of C60 with a Mixed [3:2] Octahedral Addition Pattern, Eur. J. Org. Chem. 2018, 25792586.

[9] E. Castro, K. Azmani, A. H. Garcia, A. Aghabali, S. Liu, A. J. Metta-Magana, M. M.

Olmstead, A. Rodríguez-Fortea, J. M. Poblet and L. Echegoyen, (2017), Unusual C2h-

Symmetric trans-1-(Bis-pyrrolidine)-tetra-malonate Hexa-Adducts of C60: The Unexpected Regio- and Stereocontrol Mediated by Malonate-Pyrrolidine Interaction, Chem. Eur. J. 23, 15937-15944.

[10] W. Yan, S. M. Seifermann, P. Pierrat and S. Brase, (2015), Synthesis of highly functionalized C60 fullerene derivatives and their applications in material and life sciences, Org. Biomol. Chem. 13, 25-54.

[11] F. Beuerle, N. Chronakis and A. Hirsch, (2005), Regioselective synthesis and zone selective deprotection of [60]fullerene tris-adducts with an e,e,e addition pattern, Chem. Commun. 36763678.

[12] R. Schwenninger, T. Müller and B. Kräutler, (1997), Concise Route to Symmetric Multiadducts of [60]Fullerene: Preparation of an Equatorial Tetraadduct by Orthogonal Transposition, J. Am. Chem. Soc. 119, 9317-9318.

[13] L. Isaacs, F. Diederich and R. F. Haldimann, (1997), Multiple Adducts of C60 by TetherDirected Remote Functionalization and synthesis of soluble derivatives of new carbon allotropes Cn(60+5), Helv. Chim. Acta 80, 317-342.

[14] L. Isaacs, R. F. Haldimann and F. Diederich, (1994), Tether-Directed Remote

Functionalization of Buckminsterfullerene: Regiospecific Hexaadduct Formation, Angew. Chem. Int. Ed. Eng. 33, 2339-2342.

[15] C. García-Simón, M. Garcia-Borràs, L. Gómez, T. Parella, S. Osuna, J. Juanhuix, I. Imaz, D. Maspoch, M. Costas and X. Ribas, (2014), Sponge-like molecular cage for purification of fullerenes, Nat. Commun. 5, 5557. 
[16] J.-F. Nierengarten, (2017), Fullerene hexa-adduct scaffolding for the construction of giant molecules, Chem. Commun. 53, 11855-11868.

[17] E. Castro, A. H. Garcia, G. Zavala and L. Echegoyen, (2017), Fullerenes in biology and medicine, J. Mater. Chem. B 5, 6523-6535.

[18] A. Hirsch and O. Vostrowsky, (2001), C60 Hexakisadducts with an Octahedral Addition Pattern - A New Structure Motif in Organic Chemistry, Eur. J. Org. Chem. 2001, 829-848.

[19] A. Muñoz, D. Sigwalt, B. M. Illescas, J. Luczkowiak, L. Rodríguez-Pérez, I. Nierengarten, M. Holler, J.-S. Remy, K. Buffet, S. P. Vincent, J. Rojo, R. Delgado, J.-F. Nierengarten and N. Martín, (2015), Synthesis of giant globular multivalent glycofullerenes as potent inhibitors in a model of Ebola virus infection, Nat. Chem. 8, 50.

[20] W. Krätschmer, L. D. Lamb, K. Fostiropoulos and D. R. Huffman, (1990), Solid C60: a new form of carbon, Nature 347, 354.

[21] J. Friedl, M. A. Lebedeva, K. Porfyrakis, U. Stimming and T. W. Chamberlain, (2018), AllFullerene-Based Cells for Nonaqueous Redox Flow Batteries, J. Am. Chem. Soc. 140, 401-405.

[22] S. Vidal, (2015), Sweet fullerenes vanquish viruses, Nat. Chem. 8, 4.

[23] G.-F. Liu, M. Filipović, I. Ivanović-Burmazović, F. Beuerle, P. Witte and A. Hirsch, (2008), High Catalytic Activity of Dendritic C60 Monoadducts in Metal-Free Superoxide Dismutation, Angew. Chem. Int. Ed. 47, 3991-3994.

[24] C.-H. Chiang, M. K. Nazeeruddin, M. Grätzel and C.-G. Wu, (2017), The synergistic effect of $\mathrm{H} 2 \mathrm{O}$ and DMF towards stable and $20 \%$ efficiency inverted perovskite solar cells, Energy Environ. Sci. 10, 808-817.

[25] D. Canevet, E. M. Pérez and N. Martín, (2011), Wraparound Hosts for Fullerenes: Tailored Macrocycles and Cages, Angew. Chem. Int. Ed. 50, 9248-9259.

[26] C. P. Ioannou and N. Chronakis, (2013), The first one-pot synthesis of a chiral pentakisadduct of C60 utilising an opened-structure malonate tether, Chem. Commun. 49, 10611-10613. [27] W. Brenner, T. K. Ronson and J. R. Nitschke, (2017), Separation and Selective Formation of Fullerene Adducts within an MII8L6 Cage, J. Am. Chem. Soc. 139, 75-78.

[28] B. Chen, J. J. Holstein, S. Horiuchi, W. G. Hiller and G. H. Clever, (2019), Pd(II) Coordination Sphere Engineering: Pyridine Cages, Quinoline Bowls, and Heteroleptic Pills Binding One or Two Fullerenes, J. Am. Chem. Soc. 141, 8907-8913.

[29] N. Huang, K. Wang, H. Drake, P. Cai, J. Pang, J. Li, S. Che, L. Huang, Q. Wang and H.-C. Zhou, (2018), Tailor-Made Pyrazolide-Based Metal-Organic Frameworks for Selective Catalysis, J. Am. Chem. Soc. 140, 6383-6390.

[30] G. Bottari, O. Trukhina, A. Kahnt, M. Frunzi, Y. Murata, A. Rodríguez-Fortea, J. M. Poblet, D. M. Guldi and T. Torres, (2016), Regio-, Stereo-, and Atropselective Synthesis of C60 Fullerene Bisadducts by Supramolecular-Directed Functionalization, Angew. Chem. Int. Ed. 55, 11020-11025.

[31] Y. Xu, R. Kaur, B. Wang, M. B. Minameyer, S. Gsänger, B. Meyer, T. Drewello, D. M. Guldi and M. von Delius, (2018), Concave-Convex $\pi-\pi$ Template Approach Enables the Synthesis of [10]Cycloparaphenylene-Fullerene [2]Rotaxanes, J. Am. Chem. Soc. 140, 1341313420.

[32] F. J. Rizzuto, L. K. S. von Krbek and J. R. Nitschke, (2019), Strategies for binding multiple guests in metal-organic cages, Nat. Rev. Chem. 3, 204-222.

[33] F. Djojo, A. Hirsch and S. Grimme, (1999), The Addition Patterns of C60 Trisadducts Involving the Positional Relationships e and trans-n $(n=2-4)$ : Isolation, Properties, and 
Determination of the Absolute Configuration of Tris(malonates) and Tris[bis(oxazolines)], Eur. J. Org. Chem. 1999, 3027-3039. 